\title{
CAPACITAÇÃO DE TUTORES PARA ESTRATÉGIAS DE APRENDIZAGEM ATIVA NAS ÁREAS DE STEM
}

Arthur R. Costa - arreico@gmail.com

Filipe A. Batista-filipe.aziz@gmail.com

Larissa P.C. Santos - larissapsanntos@gmail.com

Marcus J. A. Oliveira-marcus.jesse1@gmail.com

Dianne M.Viana-diannemv@unb.br

Jones Y. M. A. Silva-jonesyudi@unb.br.com

Faculdade de Tecnologia

Emily M. B. Cornelio-emilly.mbcornelio@gmail.com

Faculdade do Gama, Campus Gama

Fernanda R. Silva-fernandarose27@gmail.com

Faculdade de Educação

Carla M.C.C.Koike-ckoike@unb.br

Instituto de Ciências Exatas, Departamento de Ciência da Computação

Universidade de Brasília

Campus Darcy Ribeiro, L3 Norte

70910-900 - Brasília - DF

Resumo: Recentemente, percebe-se um aumento no número de trabalhos que abordam estratégias e métodos de aprendizagem ativa na área de educação em engenharia, relatando experiências realizadas em sala de aula. No entanto, poucas experiências abordam a importância do tutor, estudante de graduação, e de sua capacitação como facilitador nessas estratégias. $O$ intuito deste trabalho é apresentar o planejamento de uma oficina de programação de microcontroladores que futuramente será aplicada para estudantes do Ensino Médio como parte de um projeto que estimula a universalização do aprendizado e a escolha de uma profissão nas áreas de STEM (Ciências, Tecnologia, Engenharia e Matemática). Estratégias visando ao aprendizado autônomo e atividades "hands on" são coadjuvantes do ensino-aprendizagem de programação com Arduino, introduzindo-se conceitos de circuitos, componentes eletrônicos e linguagens de programação. Na oficina final planeja-se que estudantes de nível médio tenham a oportunidade de escrever algoritmos, codificá-los em linguagem de programação, montar circuitos e conectá-los à plataforma Arduino, assim como testar o funcionamento. Neste trabalho é apresentada e discutida uma execução teste dessa oficina que foi aplicada em um grupo de graduandos como parte de um treinamento de tutoria, em que estes assumem o papel dos aprendizes. Reflexões acerca dos beneficios no uso de metodologias de aprendizagem ativa para incentivar o interesse e a curiosidade dos alunos em sala de aula e do papel do tutor nesse processo, serão discutidas.

Palavras-chave: Oficina pedagógica, STEM, Programação com Arduino. 


\section{INTRODUÇÃO}

É evidente a importância do papel do tutor na educação. Diversos estudos mostram que este é um elo relevante no que diz respeito à gestão do conhecimento, por isso, a capacitação de tutores deve envolver várias dimensões da aprendizagem: interação, cooperação, cognição, metacognição e afeto (MEDEIROS e FARIA, 2003). Neste trabalho, adicionamos uma nova dimensão à formação de tutores: a utilização de metodologias de aprendizagem ativa.

Segundo Felder e Brent (2009) a aprendizagem ativa é tudo relacionado à disciplina ministrada que não seja uma aula onde os estudantes apenas assistem passivamente. A aprendizagem ativa é a aprendizagem que se constrói com debates, criação de protótipos ou projetos, experiências, resolução de problemas em grupos dentre outras atividades.

Uma dessas metodologias ativas é a atividade prática, "mão na massa", ou hands on activity, a qual se caracteriza pelo aprendizado adquirido por meio da experiência (HOLSTERMANN et al., 2009), o estudante tem a oportunidade de criar algo e aprender durante o processo de desenvolvimento. Com as atividades hands on, a aprendizagem se torna lúdica, realística e prazerosa tendo o potencial de influenciar positivamente os interesses de estudantes nas áreas de STEM. Um dos exemplos de atividade hands on utilizada durante a oficina de programação com Arduino, é a criação de protótipos a partir de projetos.

Um outro método é o flipped classroom, traduzido como sala de aula invertida. De acordo com Schmidt e Ralph (2016), nela o professor inverte o roteiro comumente utilizado nas aulas tradicionais: inicialmente uma aula conteudista, a seguir a resolução de alguns exercícios e por último a recomendação de umas poucas atividades onde é esperado que aluno apenas repita o que o professor fez durante a fase de resolução de exercícios. Na aula invertida o estudante deve estudar conteúdos antes da aula, a fim de que a aula em si se torne um espaço voltado para a elucidação de dúvidas, debates e, inclusive, atividades hands on.

Em uma pesquisa feita nos EUA com 58 professores atuantes na educação primária, matemática e ciências sociais no ensino médio, com diferentes tempos de experiência na profissão, Schmidt e Ralph (2016) puderam constatar um número escasso de professores que aderem à aula invertida. Dos 58 professores apenas 3 utilizavam tal método. Entretanto, apesar de serem poucos, estes professores admitiram um engajamento maior por parte dos alunos, menos tarefas incompletas e inclusive notas maiores, mostrando que tal metodologia é eficaz quando se trata de ajudar estudantes no processo de aprendizagem.

Constata-se a importância de se utilizar metodologias de aprendizagem ativa como um dos meios capazes de incentivar o estudante na consolidação de conhecimentos. Tal fato pode ser verificado nas experiências já relatadas no ponto de vista do professor em experiências de sala de aula, mas pouco se enfatiza na formação do estudante tutor.

Nesse sentido, o objetivo principal do trabalho é apresentar o planejamento de uma oficina de programação de microcontroladores, utilizando metodologias de aprendizagem ativa, e também, fazer uma reflexão acerca da capacitação de estudantes de graduação ao exercerem o papel de tutores.

Em uma aplicação-teste, os tutores realizaram a oficina para outros estudantes de graduação que assumiram o papel de aprendizes. Os estudantes aprendizes, nesse caso, desempenharam o papel dos estudantes de ensino médio, público alvo da oficina proposta. E em uma oportunidade futura atuarão como tutores nas oficinas para o ensino médio. Este processo de capacitação de tutores faz parte de um projeto que estimula o interesse nas áreas de STEM.

Este artigo foi estruturado como a seguir. Primeiramente, será abordado o papel do tutor de STEM no emprego das metodologias ativas. Logo, destaca-se os procedimentos metodológicos utilizados, como se deu o planejamento da oficina de programação com 
"Os desafios para formar hoje o engenheiro do amanhã"

Arduino e a execução da oficina. Posteriormente, aborda-se o os resultados e discussões obtidos através da aplicação de questionários para tutores e aprendizes.

\section{O PAPEL DO TUTOR DE STEM NO EMPREGO DA APRENDIZAGEM ATIVA}

Ao longo da história da educação, várias tendências pedagógicas surgiram como forma de encontrar, dentre outras coisas, metodologias eficazes no processo de ensino-aprendizagem de estudantes.

Atualmente, com a era tecnológica a qual o mundo está vivenciando, várias metodologias estão em evidência e dentre elas a STEM. A STEM faz parte das chamadas metodologias ativas, que se caracterizam por estimular processos construtivos de ação-reflexão-ação (FREIRE, 2006) e as quais surgiram a partir do ensino híbrido, ou blended-learning, que se caracteriza por parte do aprendizado em um programa educacional formal ocorrer online. (HORN e STAKER, 2015).

Nesta perspectiva, Freeman et al. (2014), em uma pesquisa com mais de 225 estudos diversos nas áreas de STEM, concluíram que a utilização de métodos de aprendizagem ativa resultaram em uma melhora significativa no desempenho acadêmico dos estudantes, aumentando assim sua autoestima e diminuindo a evasão, o que ressalta a importância do tema do presente trabalho.

Apesar de trazer benefícios, alguns estudantes podem ser resistentes ao emprego de metodologias ativas em suas aulas devido ao costume com o método tradicional e conteudista de aula. Entretanto não se deve ceder à tais resistências, mas usar diferentes métodos para aplicar com sucesso a aprendizagem ativa. Como exemplo, Schmidt e Ralph (2016) ao entrevistar 3 professores que utilizam a aula invertida perguntam quais estratégias usam para que o aluno tenha contato prévio com os assuntos ministrados em aula. As respostas variaram desde atividades online onde o aluno deve assistir vídeos e responder questionários até a leitura do livro didático onde o aluno deve sublinhar em diferentes cores pontos chaves, novo vocabulário e conceitos confusos.

Outra estratégia é o uso de exemplos práticos, relevantes e familiares aos estudantes. Srinath (2014) afirma que em aulas de STEM, os alunos devem aprender sobre física e matemática a fim de conseguir aplicar tais conhecimentos posteriormente. Logo, para que os alunos absorvam o máximo de conceitos explicados em sala de aula, exemplos de situações familiares aos alunos devem ser tratados juntamente com a teoria para motivar o estudo e facilitar a visualização e conexão com a teoria, o que consequentemente melhorará a aprendizagem.

Ademais o próprio estudante ao atuar como tutor consolida o conhecimento por meio da aprendizagem ativa. Nesse método, o estudante deve revisar ou aprender conceitos a fim de explicá-los a outros alunos além de sanar dúvidas, fazendo com que seu conhecimento seja posto à prova. Srinath (2014) explica que a ideia por trás desta técnica é que se o estudante conseguir explicar um conceito a outra pessoa, então ele de fato entende o conceito.

Isto posto, entende-se que a tutoria associada à autonomia na organização da aprendizagem, estimula a compreensão e favorece melhores condições para os estudantes aprenderem (FRISON, 2012). Além de que, ao ensinar, o estudante-tutor aprende enquanto interage com o aprendiz, prática que valoriza a autonomia e estimula a troca de saberes, aspectos estes essenciais na aprendizagem ativa. Cabe mencionar que a tutoria enseja "o desenvolvimento integral dos estudantes, nas suas dimensões intelectual, afetiva, pessoal e social" (SIMÃO et.al, 2008, p. 77). 


\section{METODOLOGIA}

Como proposta de capacitação de tutores, 3 professores dos cursos de engenharia mecânica e mecatrônica orientaram uma equipe de 5 estudantes de graduação e 1 estudante de pedagogia para atuarem em projetos de extensão desenvolvendo, executando e aprimorando oficinas STEM para estudantes de Ensino Médio.

Apoiou-se em estreita associação na qual os participantes representativos da situação, estudantes tutores e aprendizes, se envolveram de forma cooperativa e colaborativa para o desenvolvimento da oficina, cujo tema foi proposto pelos próprios estudantes.

Cabe aqui definir os papéis desempenhados pelos estudantes de graduação. O estudante tutor é aquele que se envolve com o desenvolvimento (elaboração e aplicação) da oficina. $\mathrm{O}$ estudante aprendiz é aquele que realiza a oficina, testando-a no lugar do estudante de Ensino Médio e, por conseguinte, é aquele que replicará a oficina posteriormente para os estudantes de Ensino Médio. Dessa forma, tanto o estudante tutor quanto o estudante aprendiz são capacitados durante a ação de desenvolvimento da oficina, embora sob diferentes pontos de vista e para diferentes objetivos.

Para a realização da oficina, todos os tutores devem ter domínio sobre eletrônica básica e seus conceitos, sobre diversos componentes eletrônicos, sobre programação básica em linguagem $\mathrm{C}$ e sobre a placa Arduino e o software utilizado em conjunto com ela.

O estudante no papel de tutor deverá adquirir o domínio das habilidades técnicas previamente ou durante a preparação da oficina. O estudante no papel de aprendiz deverá desenvolver tais habilidades durante a execução da oficina. Além das habilidades e competências técnicas, são trabalhadas a expressão oral, trabalho em equipe, a empatia, análise crítica e reflexiva, observação.

No caso do aprendiz, em particular, a aplicação desse método possibilita que os estudantes de graduação possam, ao vivenciar a experiência de aprendiz, entender melhor como os estudantes de Ensino Médio receberão as explicações e atividades, e avaliar o resultado de cada método e recurso didático utilizados.

Assim, são definidas 3 fases para o desenvolvimento da oficina e capacitação dos tutores: (i) planejamento; (ii) execução e (iii) análise dos resultados; as quais são detalhadas a seguir. Como instrumento para análise dos resultados foram utilizados questionários e observados os desempenhos dos tutores e aprendizes durante a execução da oficina, sendo realizada uma análise qualitativa.

\section{PLANEJAMENTO DA OFICINA DE PROGRAMAÇÃO COM ARDUINO}

Ao se proceder o planejamento de uma oficina educativa, é preciso em primeiro lugar determinar o objetivo a ser cumprido com a aplicação da oficina para a seguir determinar os passos que devem ser tomados e como devem ser realizados.

Para a oficina em questão, foi determinado que o objetivo final seria capacitar os aprendizes para a criação autônoma de programas em Arduino.

Inicialmente foram definidas as habilidades que os aprendizes necessitam para que sejam capazes de utilizar a placa Arduino para acionar e controlar a velocidade angular de um motor, as quais são: compreensão e uso do código de programação para a placa processar; compreensão acerca dos componentes eletrônicos a serem usados e sobre o funcionamento do circuito; habilidade manual e cognitiva para montar o circuito eletrônico a ser controlado pela placa e o código criado. A partir da definição das habilidades e tendo noção dos conhecimentos prévios esperados dos aprendizes finais (estudantes do Ensino Médio), foi possível determinar quais conteúdos devem ser abordados e compreendidos pelos aprendizes 
(estudantes de graduação e tutores dos estudantes do Ensino Médio) durante a oficina. A seguir elaborou-se o plano de aula referente a essa oficina.

Estipulou-se um tempo máximo para a realização da oficina de $3 \mathrm{~h}$ e estabeleceu-se que ela seria dividida em duas etapas. Na primeira etapa, com duração de 30 minutos, os conhecimentos teóricos necessários seriam apresentados aos aprendizes e, na segunda etapa, com duração de $2 \mathrm{~h}$ e 30 minutos seriam realizadas atividades práticas (também chamadas de hands on) para consolidação dos conhecimentos adquiridos.

Sendo assim, para que fosse possível ajustar as etapas planejadas ao tempo estipulado anteriormente, foi necessário realizar um estudo dos tempos necessários para cada atividade prático e a respectiva explicação teórica. Dessa forma, uma lista de assuntos para a explicação teórica e uma lista de exercícios práticos foram organizadas.

O passo seguinte consistiu no envolvimento de toda a equipe (estudantes tutores, coordenadores e colaboradores) na avaliação do planejamento inicial da oficina para realização de possíveis ajustes. Nesta fase alguns alunos se encarregaram de testar as atividades de programação

Em relação à explicação teórica, foi discutida a importância de cada tópico e a profundidade que cada assunto deveria ter. Observou-se que não era possível se ter certeza da área de domínio prévio dos aprendizes, dessa forma, foi prevista a aplicação de um questionário ao início da oficina com o objetivo de avaliar os conhecimentos prévios dos aprendizes e assim concentrar-se na explicação conteúdos não dominados ainda pelos aprendizes. Esse questionário poderia ser elaborado na plataforma online Kahoot!, o que possibilitaria que os aprendizes pudessem respondê-lo utilizando seus smartphones, seus computadores ou ainda os computadores presentes na sala de aula. Devido à incerteza do tempo exato que levaria a explicação da parte teórica, foi prevista uma folga.

Em relação à parte prática, de atividades hands on, foram discutidos os seguintes pontos: a relevância e dificuldade de cada atividade, e a variedade do conjunto de atividades. Definiuse também os componentes a serem utilizados como LEDs, motores, botões, alguns sensores, resistores e outros. Como o tempo que seria utilizado na explicação teórica apresentava uma margem de variação, foram criadas atividades extras para o caso de ter sobra de tempo e os aprendizes se motivassem em realizá-los. Para determinar o tempo aproximado com a realização das atividades hands on foram realizados testes com três voluntárias estudantes de graduação (duas de engenharia mecânica e uma de pedagogia) e os resultados foram utilizados para realizar ajustes nos exercícios.

Dado que a oficina seria aplicada a um grupo de graduandos como parte de uma capacitação extrínseca de tutoria, viu-se o benefício de se realizar uma discussão e debate ao fim da oficina, entre todos os envolvidos, incluindo os aprendizes, com o objetivo de avaliar os pontos positivos e negativos da oficina após a sua execução para os aprendizes, e assim poder aprimorar a oficina para ser aplicada ao Ensino Médio. Para auxiliar ainda mais na avaliação da oficina, foram aplicados questionários via Google Forms, um para os tutores e outro para os aprendizes, para que pudessem avaliar individualmente vários aspectos da oficina.

\section{EXECUÇÃO DA OFICINA}

Para a fase de execução da oficina, foi utilizado um laboratório de informática padrão: projetor multimídia, quadro branco e estações de trabalho com computadores individuais. A oficina foi realizada seguindo as etapas já explicadas.

As explicações abordaram conceitos básicos da eletrônica como corrente elétrica, tensão elétrica, resistência e Lei de Ohm. Explicou-se também o funcionamento de alguns componentes eletrônicos - como fonte, protoboard, resistor, potenciômetro, LED, botões e 
motores -, e explorou-se a computação e programação com foco no básico da linguagem C e introdução ao Arduino.

A seguir foram distribuídos kits com uma placa Arduino e alguns componentes eletrônicos, os quais foram abordados na parte teórica. Cada aprendiz pôde usar um computador do laboratório para que fosse possível praticar os exercícios individualmente. Utilizando o projetor, o tutor responsável apresentou as atividades a serem realizadas juntamente com os componentes eletrônicos que deveriam ser utilizados em cada atividade. Algumas atividades foram feitas com o acompanhamento do tutor, que demonstrou a montagem da parte eletrônica e quais códigos deveriam ser escritos. De forma intercalada, outras atividades foram propostas, para que os aprendizes realizassem de forma autônoma, tanto a montagem da parte eletrônica como a digitação do código do Arduino. Eventualmente poderiam tirar dúvidas com os tutores ou ter auxílio de outros aprendizes. Durante a oficina, o tutor responsável deixou de apresentar os códigos em páginas de slides, passando a digitar os códigos, mostrando na tela projetada, juntamente com os aprendizes.

Por fim, na última etapa da oficina, foi permitido que os aprendizes manipulassem o Arduino e os componentes eletrônicos como desejassem, podendo criar circuitos e códigos para o Arduino como quisessem, dessa forma exercitando a criatividade e os conhecimentos adquiridos durante a oficina.

\section{ANÁLISE DOS RESULTADOS E DISCUSSÕES}

\subsection{Oficina}

Durante a oficina foi possível observar diversos aspectos do desenvolvimento tanto dos aprendizes como dos tutores, bem como a dinâmica da oficina. Sendo assim, foi feito um debate com os envolvidos na oficina, incluindo alguns dos aprendizes, detalhando pontos positivos e negativos desta.

Inicialmente, ficou evidente o descumprimento de alguns tópicos abordados no plano de aula criado. Um deles foi a ausência de um material a ser enviado aos alunos no dia anterior à oficina, caracterizando uma aula invertida (flipped classroom). A falta desse material impossibilitou a aplicação do questionário previsto para ser elaborado no Kahoot! e planejado para o início da oficina, o qual também não foi realizado devido ao tempo escasso entre a preparação da oficina e sua aplicação.

Outra falha em relação ao planejamento inicial, foi a ausência da discussão sobre os resultados da oficina a serem realizados no final entre tutores, aprendizes e orientadores, a qual, foi realizada posteriormente. Também, observou-se que seria interessante acrescentar um debate ao final da oficina quando esta for realizada com os estudantes do Ensino Médio a fim de discutir a experiência que obtiveram.

Foram discutidos também os problemas técnicos ocorridos durante a oficina. Durante a etapa prática, o software IDE gerou falhas e problemas para alguns aprendizes, tais como, o não reconhecimento das placas Arduino e a falha no envio do código escrito à placa. Para contornar esses problemas o funcionamento do Arduino deveria ter sido testado previamente em cada computador. Ainda, foram constatados alguns problemas relacionados à didática. Observou-se que, durante as explicações teóricas, o tutor responsável não avisou aos aprendizes que poderiam tirar suas dúvidas a qualquer momento e também não deixou um momento livre para dúvidas e questionamentos, possivelmente prejudicando o entendimento dos aprendizes. Percebeu-se também que a mudança de método durante a parte prática foi positiva, os aprendizes compreenderam melhor o significado das linhas de código criadas quando o tutor responsável as digitou junto com eles ao invés de apenas explicar as linhas já escritas em páginas de slides. 
Por fim, ao final da oficina foi possível perceber que o objetivo foi cumprido com êxito. Os aprendizes conseguiram assimilar relativamente bem as explicações teóricas, conseguiram colocar em prática as atividades propostas e puderam compreender os conceitos teóricos aplicados na prática. A aprendizagem ativa de fato ocorreu, isto é, os aprendizes se envolveram em experiências significativas e conseguiram resolver problemas de forma autônoma, por meio de atividades hands on.

\subsection{Questionários}

Após a oficina de Arduino, foram aplicados dois questionários via Google Forms (um para tutores e outro para os aprendizes) com o objetivo de fazer uma reflexão acerca das estratégias e métodos adotados por tutores e o papel destes nessas práticas tendo como base os métodos e estratégias de aprendizagem ativa, bem como a ótica dos aprendizes em relação a tais aspectos.

Diante disso, quando perguntados acerca de como avaliam o uso de metodologias de aprendizagem ativa na educação, teve-se uma resposta bastante positiva. Em relação à postura dos aprendizes acerca da aplicação desses mesmos métodos e estratégias de aprendizagem ativa, ou seja, se estes foram participativos ou não, a resposta também foi positiva.

Os tutores quando questionados acerca dos conhecimentos adquiridos (Figura 1), temos aspectos como: aprendeu a ensinar de forma lúdica e didática, a se colocar no lugar do outro, a fazer uso de atividades práticas, a importância de se ter um bom domínio do conteúdo, recursos e ferramentas pedagógicas.

Figura 1 - Aprendizado adquirido pelos estudantes enquanto tutores

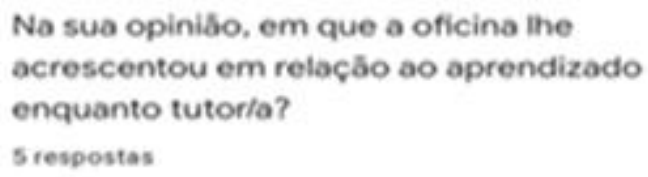

Me acrescentou em como é importante ensinar um conteudo novo de forma ludica (no caso das ifustraços e das cores dos elementos utilizados, que deixaram o entendimento das atividades mais facil)

Me coloquei no lugar do aluno, e pude ver quais pontos posso melhorar enquanto tutora.

o fato da oficina ser aplicada com os tutores antes de ser aplicada com as alunas do ensino médio nos ajudou a entender que algumas coisas precisam ser explicadas de outro jeito, de um jeito mais didático. a oficina tbm ajudou com a atividade prática que ajudou até mesmo os alunos que jo tiveram contato com robotica a esclarecer dôvidas.

\begin{abstract}
antes de ser aplicada com as alunas do ensino médio nos ajudou a entender que algumas coisas precisam ser explicadas de outro jeito, de um jeito mais didatico, a oficina tbm ajudou com a atividade prática que ajudou até mesmo os alunos que já tiveram contato com robótica a esclarecer dúvidas.
\end{abstract}

Aprender melhor os recursos para serem passados. Pois como me diverti bastante acredito com uma boa dominação no contećdo será bem interessante meu papel como tutor

Entender como aplicar as ferramentas, que sáo super interessantes, com alunos de ensino medio, que costumam se entediar facil.

Fonte: Os autores.

Apesar de as metodologias de aprendizagem ativa serem consideradas benéficas para o aprendizado e conseguirem manter os alunos participativos, o tutor encontrou dificuldades na realização do planejamento de aula da oficina, em relação à adaptar o linguajar técnico ao público alvo que seria o do Ensino Médio, ou seja, de torná-lo mais acessível, o que implicou 
também, em uma nota auto avaliativa numa escala de 0 a 10 , de nota 5 , ou seja, não muito satisfeito consigo mesmo neste quesito.

Em relação a aspectos que precisam ser melhorados, obteve-se como resposta a didática, a qual para Veiga (1989, p. 44), "é compreendida como um conjunto de regras visando assegurar aos futuros professores as orientações necessárias ao trabalho docente."

Quanto ao questionário aplicado aos aprendizes, quando indagados se aulas que utilizam metodologias ativas auxiliam no melhor entendimento sobre os assuntos, teve-se por unanimidade que sim, revelando que estas resultam em um envolvimento autônomo por parte do estudante na busca pelo conhecimento.

Por fim, já no que diz respeito ao fato da oficina ter relação com as práticas cotidianas e isso ajudar no processo de aprendizagem, tem-se o percentual de $100 \%$, ou seja, a educação precisa fazer sentido e não ser algo desvinculado da realidade social dos educandos e educandas, logo, o currículo escolar precisa "contextualizar os conteúdos dos componentes curriculares, identificando estratégias para apresentá-los, representá-los, exemplificá-los, conectá-los e torná-los significativos, com base na realidade do lugar e do tempo nos quais as aprendizagens estão situadas.” (BNCC, 2015, p. 16).

\section{CONSIDERAÇÕES FINAIS}

Diante do exposto, pode-se concluir que, a oficina de capacitação de tutores para estratégias de aprendizagem ativa e programação com Arduino foi exitosa, mesmo com as dificuldades encontradas ao longo do planejamento e execução desta, já que os objetivos propostos para a oficina foram alcançados.

É de conhecimento comum que competências quanto à noção de assuntos de programação e eletrônica se fazem importantes na presente era digital. Seu ensino desperta o interesse, a curiosidade e a motivação dos estudantes na aprendizagem de disciplinas relacionadas ao assunto como física e matemática que muitas das vezes são alvo de rejeição pela maioria de estudantes da educação básica no Brasil.

Sendo assim a oficina Programação com Arduino se mostra relevante na tarefa, muitas vezes difícil, de incentivo a estudo nas áreas de ciências exatas. Além do mais, percebe-se que, o Arduino, com seu cunho lúdico e prático, aliado às metodologias ativas no ensino desta ferramenta de prototipagem eletrônica, se faz um ótimo meio para a apresentação de novos assuntos quanto à tecnologia e engenharias podendo servir de estímulo para que estudantes ainda do ensino médio cogitem seguir carreira nessas áreas.

Quanto aos tutores, o planejamento da oficina até sua fase de aplicação se mostrou de grande potencial para pôr em prática habilidades já aprendidas em seus cursos de graduação ou ainda pra aprender assuntos ainda não abordados em sala de aula. Ao atuar como tutor o aluno de graduação se mantém ativo em sua aprendizagem, deixando de lado a passividade do método tradicional de ensino-aprendizagem frequentemente usado em aulas. Além do mais, como mostrou os questionários, o uso de metodologias ativas foi bem avaliado pelos tutores, mostrando-se uma abordagem divertida para o estudo.

Ademais, além dos questionários mostrarem uma resposta positiva quanto ao processo de ensino-aprendizagem proporcionado pela junção de metodologias ativas junto à tutoria, respostas de aprendizes nesses mesmos questionários mostram que apesar da aprendizagem ter se dado de forma lúdica, didática e exemplos da vida cotidiana terem os auxiliado na aprendizagem, houve uma dificuldade por parte dos tutores para que os mesmos deixassem de usar a linguagem técnica universitária quanto à alguns conceitos, situação essa que deve ser mudada e melhorada para que futuramente alunos do ensino médio não encontrem dificuldades quanto a isso. 
Por fim, a oficina descrita utilizou uma placa de prototipagem Arduino para a montagem de circuitos. Entretanto, como alternativa para casos onde não há uma placa igual ou semelhante disponível, recomenda-se o uso da plataforma Tinkercad que simula a montagem de circuitos em uma placa Arduino. Apesar de limitante, esta mesma plataforma foi utilizada em momentos específicos da oficina pelo tutor responsável, mostrando-se eficaz em termos de ilustração e simulação dos circuitos.

Com os resultados obtidos, a oficina será aprimorada para posterior aplicação final com alunos do Ensino Médio e novas oficinas serão criadas. Destaca-se a relevância deste trabalho para a área de Educação em Engenharia, bem como o fato de que potenciais trabalhos serão realizados futuramente. Espera-se, portanto, que este trabalho tenha contribuído para a reflexão do tema

\section{Agradecimentos}

À FAP-DF pelo apoio financeiro concedido por meio do Edital 03/2018 .

\section{REFERÊNCIAS}

BNCC. Ministério da Educação. Base Nacional Comum Curricular. Brasília, DF: MEC, 2015. Disponível em:

http://basenacionalcomum.mec.gov.br/images/BNCC_EI_EF_110518_versaofinal_site.pdf. Acesso em: 20 de maio de 2020.

FELDER, Richard M.; BRENT, Rebecca. Active learning: An introduction. ASQ higher education brief, v. 2, n. 4, p. 1-5, 2009.

FREEMAN, Scott et al. Active learning increases student performance in science, engineering, and mathematics. Proceedings of the National Academy of Sciences, v. 111, n. 23, p. 8410-8415, 2014.

FREIRE, Paulo. Pedagogia do Oprimido. São Paulo: Paz e Terra, 2006.

FRISON, Lourdes Maria Bragagnolo. Tutoria entre estudantes: uma proposta de trabalho que prioriza a aprendizagem. Revista Portuguesa de Educação, v. 25, n. 2, p. 217-240, 2012.

HOLSTERMANN, Nina; GRUBE, Dietmar; BÖGEHOLZ, Susanne. Hands-on activities and their influence on students' interest. Research in science education, v. 40, n. 5, p. 743-757, 2009.

HORN, Michel B.; STAKER, Heather. Blended: usando a inovação disruptiva para aprimorar a educação. Porto Alegre: Penso. 2015.

MEDEIROS, Marilú Fontoura de; FARIA, Elaine Turk (Orgs.) Educação a distância: cartografias pulsantes em movimento. Porto Alegre: EDIPUCRS, 2003.

SCHMIDT, Stacy M. P.; RALPH, David L. The flipped classroom: A twist on teaching. Contemporary Issues in Education Research (CIER), v. 9, n. 1, p. 1-6, 2016. 
"Os desafios para formar hoje o engenheiro do amanhã"

SRINATH, Adusumilli. Active Learning Strategies: An Illustrative Approach to Bring out Better Learning Outcomes from Science, Technology, Engineering and Mathematics (STEM) Students. International Journal of Emerging Technologies in Learning, v. 10, 2014.

VEIGA, Ilma P. A. A prática pedagógica do professor de didática. Campinas: Papirus, 1989.

SIMÃO, Ana M. V. et al. Tutoria no Ensino Superior: Concepções e práticas. Sísifo Revista de Ciências da Educação, 7, 75-88, 2008.

\title{
TUTOR TRAINING FOR ACTIVE LEARNING STRATEGIES IN STEM AREAS
}

\begin{abstract}
Recently, it is possible to notice a great increase in the number of papers that address strategies and methods of active learning in the field of engineering education, reporting experiences carried out in the classroom. However, few experiences address the importance of the tutor, an undergraduate student, and its training as a facilitator in these strategies. The purpose of this paper is to present the planning of a microcontroller programming workshop that will be applied to high school students as part of a project that stimulates the universalization of learning and the choice of a profession in the areas of STEM (Sciences, Technology, Engineering and Mathematics). Strategies aiming autonomous learning and hands on activities are coadjuvants of the Arduino programming teachinglearning process, introducing concepts of circuits, electronic components and programming languages. In the final workshop, it is planned that high school students will have the opportunity to write algorithms, encode them in a programming language, assemble circuits and connect them to the Arduino platform, as well as check the functionalities of the implemented hardware and software. In this paper, a test run of this workshop is presented and discussed, which was applied to a group of undergraduates as part of a tutoring training, in which they take on the role of apprentices. Reflections on the benefits of using active learning methodologies to encourage students' interest and curiosity in the classroom and the role of the tutor in this process, will be discussed.
\end{abstract}

Keywords: Pedagogical Workshop, STEM, Arduino programming. 